\title{
L'IPERMEDialità nella Didattica delle Lingue Moderne: una Ricerca
}

\author{
Anna Toscano *
}

ABSTRACT: Oggetto di questo articolo è un'indagine svolta presso alcuni istituti che insegnano lingua italiana a San Paolo nell'ambito di una ricerca che si propone di motivare l'utilizzo di un ipermedia nella didattica delle lingue moderne. L'ipermedia è indicato come uno strumento importante in ambito glottodidattico, visto che si basa su un approccio umanistico e trae ispirazione da una visione socio-costruttivista del contesto scuola. L'ipotesi di uno strumento ipermediale nella glottodidattica garantirebbe duttilità, dinamicità e apertura che potrebbe così essere usato in tutte le classi in maniera varia e differenziata. Abbiamo svolto la ricerca in tre istituti di San Paolo (Brasile) e in uno di Venezia, cercando di investigare quali possano essere i punti di contatto tra l'uso effettivo delle tecnologie in ambito glottodidattico e le preferenze di uso. Ne presentiamo qui i dati emersi nei loro punti salienti in quanto la ricerca ha portato a una raccolta di informazioni molto consistente.

PAROLE-CHIAVE: ipermedia; tecnologia; glottodidattica; ricerca; lingue moderne.

ABSTRACT: Tema deste artigo é uma investigação em algumas escolas que ensinam a língua italiana em São Paulo, como parte de uma pesquisa que tem por objetivo incentivar o uso da hipermídia no ensino das línguas modernas. A hipermídia é indicada como uma ferramenta importante no campo do ensino de línguas, já que conta com uma abordagem humanista e se inspira numa visão sócioconstrutivista do contexto escolar. A hipótese de um instrumento de ensino de língua por meio de hipermídias asseguraria flexibilidade, dinamismo e abertura, por eles poderem ser usados em aulas de todos os niveis de uma maneira diversificada e diferenciada. Realizamos nossa pesquisa em três instituições de São Paulo e uma de Veneza (Itália), tentando investigar quais poderiam ser os pontos de contato entre

\footnotetext{
* Università Ca' Foscari di Venezia (Italia) - atoscano@unive.it
} 
o uso efetivo da tecnologia no campo do ensino de línguas e as preferências de uso. Apresentamos aqui destaques dos dados coletados, já que a pesquisa levou a um conjunto de informações muito consistente.

PALAVRAS-CHAVE: hipermídia; tecnologia; ensino de línguas; pesquisa; línguas modernas.

ABSTRACT: The results of a survey on Italian language teaching at various institutes in São Paulo can be found in this article. It was carried out in the framework of a research aiming at motivating the use of a hypermedium, while teaching modern languages. The hypermedium is considered an important tool for language teaching, on the basis of a humanistic approach and because it draws inspiration from a social constructivist vision of the school context. The use of a hypermedial tool in language teaching would guarantee flexibility, dynamism and openness and could thus be used in a varied and differentiated manner. The survey was carried out at three schools in São Paulo (Brazil) and at the University of Venice (Italy), with the intent to investigate the contact points between the actual usage of technologies in language teaching and the usage preferences. Data collection in the framework of the survey is substantial, therefore only the most prominent features of data collected are presented.

KEYWORDS: hypermedium; technology; language teaching; survey; modern languages. 


\section{I presupposti della ricerca}

In un contesto umanistico della glottodidattica, oramai da decenni, l'insegnamento e l'apprendimento non sono più di tipo monomediale e accanto al libro di testo cartaceo convivono già da tempo strumentazioni diverse ${ }^{1}$. È un fatto che la didattica delle lingue ha assunto una connotazione multimediale molto prima di altri insegnamenti, ma la multimedialità della strumentazione tradizionalmente utilizzata in glottodidattica - supporti cartacei, audiovisivi, informatici - è stata affiancata in questi anni da Internet che l'ha potenziata, facendola divenire ipermedialità (ANTONELLI, 2002; LODRINI, 2002).

Multimediale e ipermediale sono due termini differenti concettualmente e tecnologicamente: multimedia indica la compresenza di più media, mentre ipermedia rimanda non solo alla compresenza di più media, ma anche all'organizzazione ipertestuale dei contenuti e alla connessione a Internet dell'insieme.

La ricerca qui presentata nasce dall'ipotesi di strutturare un ipermedia su una città, in quanto sede di una didattica esperienziale e luogo motivazionale, e sulla letteratura che alla città in questione si riferisce ${ }^{2}$. Dunque un ipermedia come strumento unico da portare in aula: non più la ricerca tra il materiale autentico per integrare il manuale di classe, ma avere al posto del manuale di classe e di tutto il materiale integrativo un unico supporto costruito con materiale autentico e che comprenda testi, immagini, video, collegamento a Internet e altro ancora, così che l'ipermedia possa anche fungere da materiale complessivo per lo studio in autonomia.

La ricerca si propone di motivare all'uso di un ipermedia, con un tema specifico, come strumento di classe al posto del libro di testo, e di fornire un percorso di insegnamento linguistico che aggreghi nuove tecnologie, nuovi apprendenti e insegnamento tradizionale.

Svolta in tre Istituti di San Paolo e in uno a Venezia, l'indagine punta a investigare quali possano essere i punti di contatto tra l'uso effettivo delle tecnologie in ambito glottodidattico e le preferenze di uso. Lo scopo dell'investigazione, al contempo, è anche quello di comprendere quale ruolo abbia la città oggi nell'apprendimento della lingua italiana (lingua seconda - L2 o lingua straniera - LS) (TOSCANO, 2012).

È stata intrapresa la strada di un'analisi qualitativa, ma anche quantitativa, basata sulla ricerca-azione (BURGESS, 1985; COONAN, 2000). In relazione ai nostri obiettivi abbiamo reputato più utile e interessante un'analisi qualitativa dei questionari, ma abbiamo proceduto anche a un'analisi quantitativa, visto il significativo numero di questionari raccolti e la rilevanza dei dati emersi.

1 Sul tema, si vedano, tra gli altri, Baldry \& Prozzo (1996), Banzato (2002), Banzato \& Corcione (2007), Ferrari (2012), Toscano (2015).

2 Sull'insegnamento della letteratura, si è usato come riferimento Acutis (1979). Questioni specifiche su educazione letteraria e nuove tecnologie sono ad esempio affrontate in un volume a cura di Balboni (2002). 
Ci siamo quindi proposti di verificare come l'uso del manuale di classe - corredato da un insieme di materiali satellite - possa essere ottimizzato attraverso la sostituzione con un ipermedia. Abbiamo anche cercato di reperire attraverso i questionari risposte concrete e precise da parte dei docenti e degli studenti sul loro approccio alla lingua, sia in contesto istituzionale sia in contesto autonomo ${ }^{3}$. Ottenute dettagliate risposte al questionario, le abbiamo utilizzate per costruire un ipermedia specifico che potesse andare a sostituire il manuale di classe.

\section{Il questionario}

Sono state elaborate due tipologie di questionari, uno per i docenti e uno per gli studenti, sostanzialmente speculari, che includono domande con la possibilità di risposte a scelta multipla e domande a risposta aperta. Abbiamo deciso di elaborare i questionari e di inviarli in forma elettronica considerando tre punti di forza di questa scelta:

- poter farli pervenire al maggior numero possibile di soggetti;

- renderli agevolmente accessibili a chi volesse partecipare;

- poter raccogliere i dati in formato elettronico.

È stato utilizzato un programma di composizione fornito dal motore di ricerca Google e i link dei rispettivi questionari sono stati inseriti in una mail accompagnatoria e inviati via mail ai destinatari selezionati. La mail è stata inviata a studenti o ex-studenti dalle rispettive segreterie degli Enti o Istituzioni contattati.

Le tabelle 1 e 2 illustrano la natura dei due questionari rispetto ai tipi di domande in essi poste.

Tabella 1: tipo di domanda nel questionario studenti

\begin{tabular}{c|c|c}
\hline Tipo di domanda & $\begin{array}{c}\text { Domande con risposte a scelta } \\
\text { multipla }\end{array}$ & Domande aperte \\
\hline Quantità & 17 & 1 \\
\hline \multicolumn{3}{c}{ Totale domande: 18} \\
\hline
\end{tabular}

Tabella 2: tipo di domanda nel questionario docenti

\begin{tabular}{c|c|c}
\hline Tipo di domanda & $\begin{array}{c}\text { Domande con risposte a scelta } \\
\text { multipla }\end{array}$ & Domande aperte \\
\hline Quantità & 13 & 1 \\
\hline \multicolumn{3}{|c}{ Totale domande: 14} \\
\hline
\end{tabular}

3 L'obiettivo era, tra l'altro, quello di riconoscere stili di apprendimento e "differenze". Sul tema si possono vedere, tra gli altri, Gardner (1993, 2005), Cadamuro (2004), Caon (2006, 2008). 


\section{Il campione}

Il focus group (MORGAN, 1997) comprende un campione che appartiene a diverse realtà. Il questionario è stato inviato a:

- studenti di Italiano L2

- docenti di Italiano L2

- studenti di Italiano LS

- docenti di Italiano LS

L'indagine è stata condotta, per quanto riguarda la L2, in un Centro Linguistico universitario, dunque a studenti adulti ${ }^{4}$. Il questionario studenti è stato inviato a coloro che hanno frequentato i corsi B1, B2, C1 e C2 nei 4 anni che hanno preceduto l'inizio della ricerca. Il questionario docenti è stato inviato ai tre CEL di italiano del Centro. Per quanto riguarda l'indagine LS, l'abbiamo condotta in tre istituti di San Paolo: l'Istituto Italiano di Cultura (IIC), l'Istituto Culturale Italo-Brasiliano (ICIB) e la scuola Spazio Italiano (SI).

L'IIC è stato fondato nel 1951 e da allora eroga corsi di Italiano (interrotti per un periodo nel 2010). Non propone corsi di altre lingue e i corsi di italiano vengono impartiti da 10 docenti per una media annuale di 300 studenti. L'ICIB è un'istituzione privata, esiste dal 1950 e da sempre eroga corsi di lingua Italiana, non ci sono corsi di altre lingue, vi insegnano 18 docenti e conta circa 2500 iscrizioni all'anno. Spazio Italiano è un'istituzione privata, fondata nel 1992, che da sempre offre corsi di lingua Italiana e talvolta corsi di lingua portoghese. I docenti erano 15 al momento della ricerca e le iscrizioni circa 360 all'anno.

I "numeri della ricerca" sono stati ricapitolati nella tabella che riportiamo di seguito:

\begin{tabular}{l|lc}
\hline \multirow{2}{*}{ Totale studenti per Istituti } & Studenti L2 CLA & 99 \\
\cline { 2 - 2 } & Studenti LS SI & 77 \\
\cline { 2 - 3 } & Studenti LS ICIB & 141 \\
\cline { 2 - 3 } & Studenti LS IIC & 2 \\
\hline \multirow{2}{*}{ Insieme totale studenti L2 e LS } & L2 & 99 \\
\cline { 2 - 2 } & LS & 220 \\
\hline Totale & & 319 \\
\hline
\end{tabular}

4 Si tratta del Centro Linguistico di Ateneo dell’Università Ca' Foscari di Venezia che è stato istituito nel 1977 e dal 2008 ha sede a San Sebastiano, sede anche della Facoltà di Lettere. 


\begin{tabular}{l|lc}
\hline \multirow{2}{*}{ Insieme totale docenti per Istituti } & Docenti L2 CLA & 3 \\
\cline { 2 - 2 } & Docenti LS SI & 4 \\
\cline { 2 - 2 } & Docenti LS ICIB & 10 \\
\cline { 2 - 2 } & Docenti LS IIC & 8 \\
\hline \multirow{2}{*}{ Insieme totale docenti per L2 e LS } & Docenti L2 & 3 \\
\cline { 2 - 2 } & Docenti LS & 22 \\
\hline Totali & & 25 \\
\hline
\end{tabular}

\section{Analisi: il questionario studenti}

Il questionario per gli studenti, dal titolo "Le strade dell'apprendimento linguistico", è consultabile a questo link e consta di vari punti. Il primo dato richiesto è la fascia d'età degli studenti ed è significativo ciò che emerge dalle risposte. Gli studenti più giovani, tra i 18 e i 24 anni, sono presenti in minor misura nel seppur vasto campione che abbiamo in esame. Reputiamo importante dare una mappatura degli studenti per collocare la loro fascia di età in quella che è l'era digitale ${ }^{5} \mathrm{e}$, in base a ciò, analizzarne i comportamenti in ambito di apprendimento delle lingue. Incrociando i dati di tutti e tre i questionari emerge che su un totale di 319 studenti adulti di lingua italiana, L2 e LS, la fascia di età maggiormente presente è quella tra i 25 e i 35 anni $(29,52 \%)$, seguita da quella compresa tra i 45 e 60 anni $(23,81 \%$, ) e, a ruota, quella tra $\mathrm{i}$ 18 e i 24 anni $(19,68 \%)$ e tra i 35 e i $45(17,46 \%)$. Chiude la percentuale di persone sopra i 60 anni $(9,52 \%)$.

Grafico 1 - Età studenti

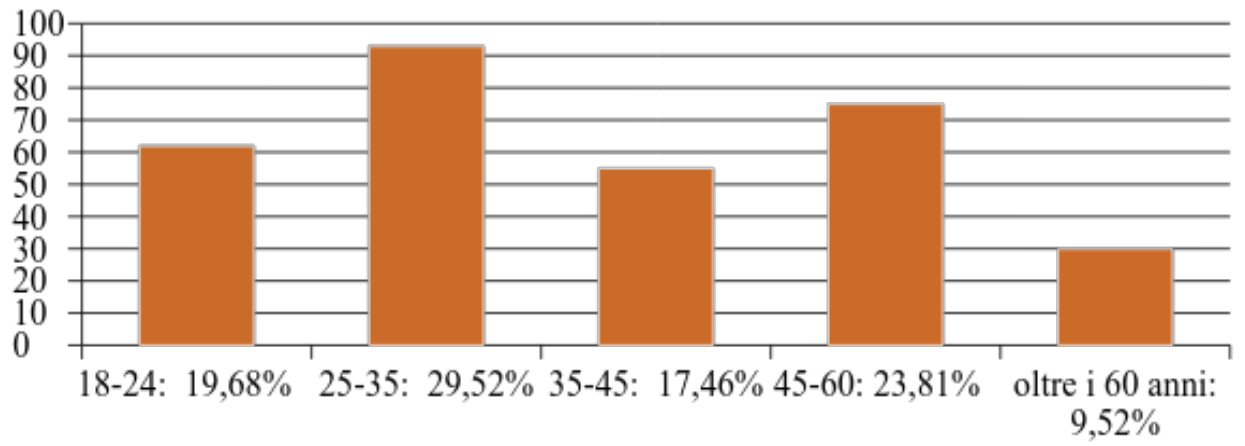

5 Sul concetto di nativo digitale, si vedano Prensky (2001) e Ferri (2011). 
Riteniamo che questo sia un dato importante in quanto dalle risposte alle altre domande abbiamo notato un sostanziale coesistere di tecnologie diverse e materiale cartaceo, sia nei percorsi istituzionali che in quelli in autonomia di apprendimento della lingua italiana. Di fatto, questo campione di studenti utilizza materiale cartaceo, Internet, video, audio quasi nella stessa percentuale e, inoltre, trova efficace l'uso di questi strumenti nei corsi di lingua tanto che nello studio autonomo rimettono in atto una simile modalità. Abbiamo unito e analizzato i dati emersi dai tre questionari alla domanda 1, riguardante quale materiale avessero utilizzato per apprendere la lingua italiana in contesti istituzionali, e ne è emersa una discreta continuità di uso: la percentuale maggiore di uso va al supporto cartaceo, come si poteva prevedere, visto l'uso largamente diffuso del manuale in quasi tutte le classi. Il materiale audio, video, Internet, cd-rom e le esperienze nella città sono tutti utilizzati in percentuali consistenti. La stessa domanda, attinente allo studio autonomo e agli strumenti che lo studente sceglie e con i quali decide di apprendere la lingua italiana in autonomia, supporta abbastanza i dati emersi dai percorsi istituzionali.

Grafico 2 - Materiale nell'apprendimento dell'italiano (percorsi istituzionali)

Nei tuoi percorsi istituzionali di apprendimento della lingua italiana quale materiale hai utilizzato:

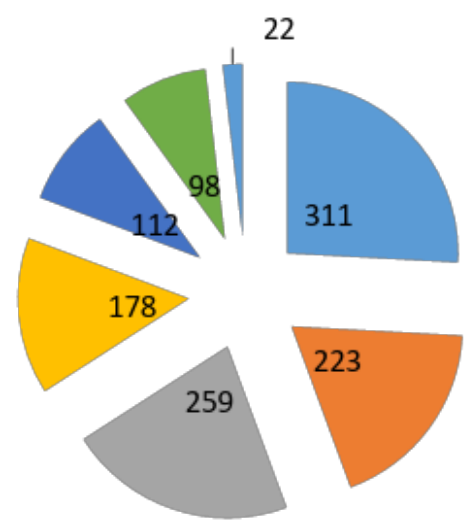

materiale cartaceo $25,85 \%$

materiale video $18,54 \%$

materiale audio $21,53 \%$

internet $14,80 \%$

cd-rom $9,31 \%$

esperienze nelle città in cui

ti trovavi $8,15 \%$

altro $1,83 \%$ 
Grafico 3 - Materiale nell'apprendimento dell'italiano (percorsi personali)

\section{Nei tuoi percorsi personali di apprendimento della lingua italiana quale materiale hai utilizzato:}

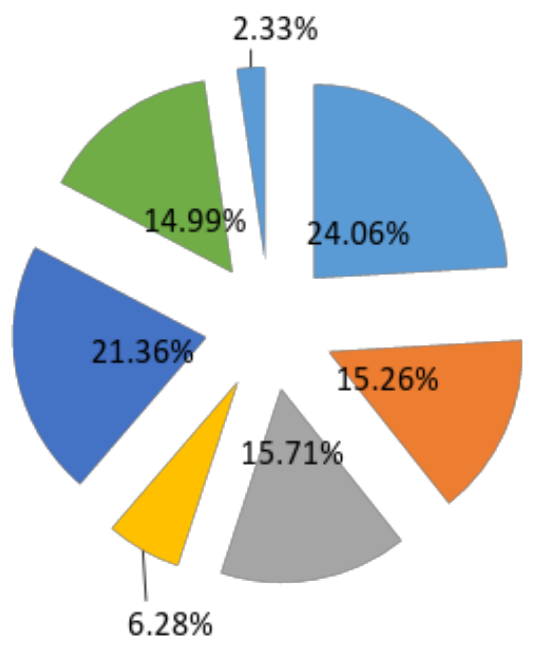

materiale cartaceo (libri,
fotocopie, ecc.)
materiale audio
materiale video
cd rom
internet
città (musei, luoghi
d'incontro, amici, ecc.
altro

Questi due grafici e la loro analisi (CLARK; LYONS, 2000) ci permettono di interpretare i dati in maniera globale: appurato l'uso di tutti questi strumenti insieme, sia nei percorsi istituzionali di apprendimento sia in quelli autonomi, il fatto di avere un unico strumento, un ipermedia, in grado di unire e raccordare questi supporti alla glottodidattica, sarebbe probabilmente una soluzione ottimale per l'insegnamento e l'apprendimento della lingua. I risultati emersi dal questionario studenti, a partire dalle domande riguardanti in modo specifico l'uso della città, ci hanno notevolmente sorpresi. Non avevamo previsto infatti una tale attenzione nei confronti della città e dei suoi "luoghi" nella prassi glottodidattica e in generale nelle fasi di apprendimento della lingua, sia in autonomia sia in percorsi istituzionali. Sottolineeremo qui la questione importante della differenza tra lingua italiana L2 e lingua italiana LS nell'uso della città. È infatti pensabile che in un contesto di italiano L2 la città nei suoi spazi possa essere utilizzata per l'apprendimento linguistico, anche perché le città italiane sono mediamente contenute in 
grandezza. Supponevamo che in contesto LS a San Paolo ci fosse una scarsa predisposizione all'uso della città per l'insegnamento/apprendimento della lingua, data anche la sua caratteristica di megalopoli. In realtà a San Paolo, come in molte altre città del mondo, la presenza artistica dell'Italia è molto viva: architettura, pittura, letteratura, cinema, cucina, ovunque ve ne sono tracce. Dunque le intersezioni con la lingua e la cultura italiana non sono affatto difficili da trovare e da strutturare in ambito glottodidattico.

La somma totale dei dati emersi dal questionario studenti dei tre istituti sull'uso della città nei percorsi istituzionali e in quelli autonomi e i dati relativi alla percezione della loro efficacia ci incoraggiano a portare avanti il nostro progetto. Infatti, come si osserva nel grafico seguente, per l'apprendimento della lingua gli studenti frequentano luoghi di incontro $(26,48 \%)$, fanno passeggiate per la città $(21,65 \%)$, altre attività fuori aula $(21,11 \%)$, visite a musei $(17,17 \%)$ e altro $(13,60 \%)$, tra cui spicca la voce "vacanze":

Grafico 4 - La città nei percorsi istituzionali

\section{Attività nella città nei percorsi istituzionali}

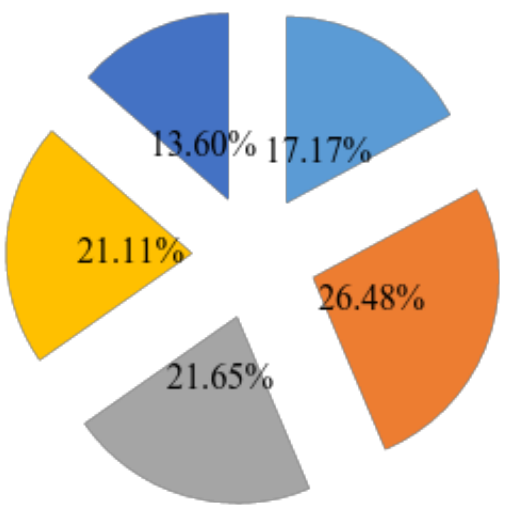

visite a musei luoghi d'incontro (bar, ristoranti, ecc.)

passeggiate per la città altre attività fuori aula altro

Infine abbiamo preso in considerazione la progettualità futura dello studente riguardo agli strumenti con cui vorrebbe continuare a imparare la lingua italiana.

Abbiamo quindi sommato i dati di tutti e tre i questionari alla domanda che indagava quali supporti lo studente avrebbe voluto usare per continuare ad apprendere la lingua italiana. La 
domanda, posta quasi a conclusione del questionario, mira a far riflettere lo studente sulle sue preferenze in virtù delle risposte appena date sul percorso di apprendimento istituzionale e autonomo esperto. Infatti in generale lo studente comprende e percepisce la soddisfazione e l'efficacia del suo percorso di apprendimento della lingua, ma spesso non riflette sul percorso glottodidattico che sta facendo. I dati che riportiamo sono, a nostro parere, indicativi in quanto marcano una grande duttilità e versatilità degli studenti. Leggiamo dal grafico la sostanziale compresenza, in simile percentuale, di diversi strumenti: materiale cartaceo $(18,23 \%)$, materiale video $(17,23 \%)$, esperienze con altri studenti (corsi di cucina, letture, visite ai musei, ecc.) $(16,23 \%)$, materiale audio $(15,46 \%)$, attività in città $(14,31 \%)$, Internet $(13,08 \%)$.

Nonostante la fascia di età maggiormente implicata non sia quella che comprende i più giovani, la predisposizione e la preferenza ad apprendere la lingua attraverso un'interazione di strumenti, dai più tradizionali come il cartaceo ai più contemporanei, è indicativa per il progetto che cerchiamo di portare avanti. L'ipermedia, genericamente, può essere strutturato con tutte le potenzialità che le diverse tecnologie offrono.

Grafico 5 - Strumenti per imparare l'italiano

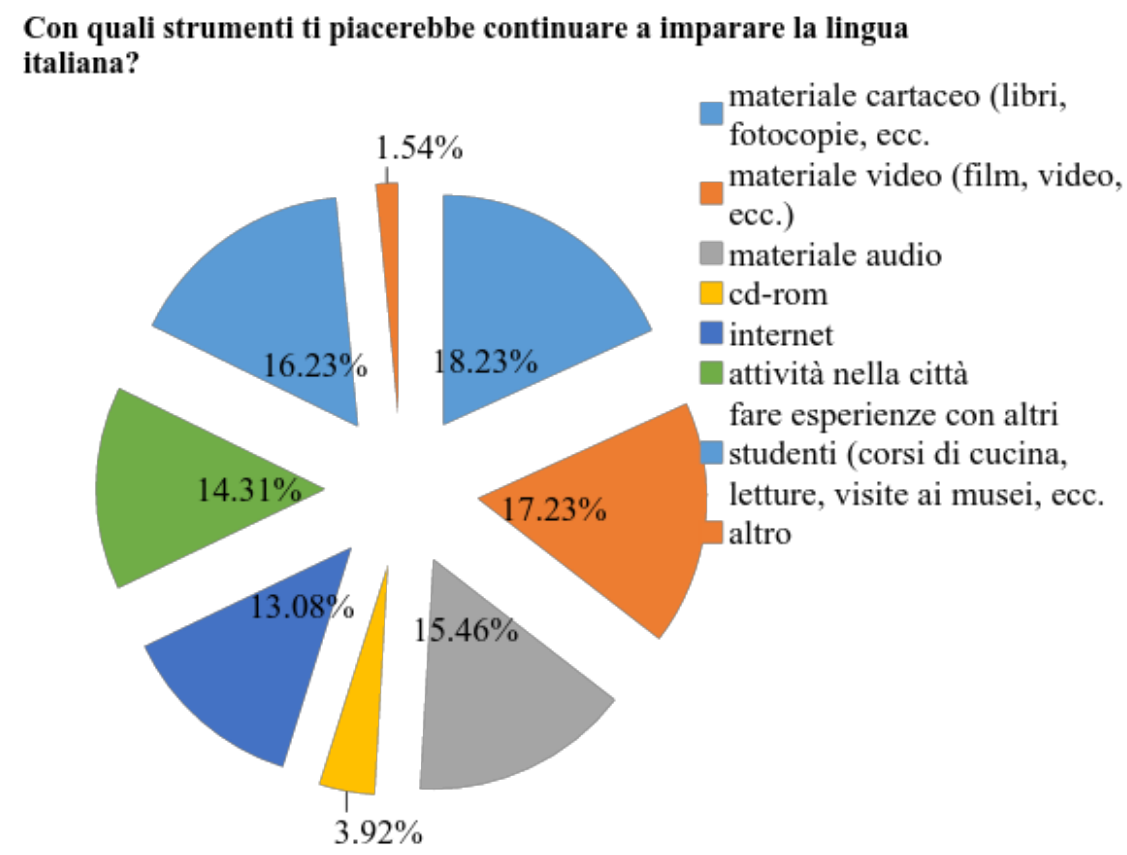




\section{Analisi dei dati del questionario docenti}

Il questionario per gli studenti, con il titolo "Le strade dell'insegnamento linguistico" e consultabile a questo link, è suddiviso in vari punti. Il primo dato richiesto riguarda l'età dei docenti. Emerge dalle risposte che l'età del maggior numero di docenti si assesta tra i 40 e i 50 anni (34,78\%); seguono coloro che hanno tra i 30 e i 40 anni $(26,09 \%)$ e chi ha tra i 50 e i 60 anni corrisponde a una percentuale più bassa $(21,74 \%)$. Solo il 13,04\% ha un'età compresa tra i 20 e i 30 anni. Sulla base di questi dati, la prima considerazione da fare è che quasi il $90 \%$ degli insegnanti di lingua italiana L2 o LS che hanno risposto al questionario sono "immigranti digitali", ovvero persone entrate nel mondo digitale in età adulta. La seconda considerazione è che l'età media dei docenti nei tre Istituti di San Paolo corrisponde all'età media degli studenti degli stessi istituti. L'unica eccezione è il CLA, i cui docenti hanno un'età media superiore a quella degli studenti. Per la nostra ricerca è importante dare una mappatura della fascia di età di appartenenza degli studenti e dei docenti che hanno risposto al questionario, per collocarla in quella che, come si è già accennato. viene denominata l'era digitale (PICHIASSI, 2007) e, in base a ciò, analizzarne i comportamenti in ambito di apprendimento e insegnamento delle lingue (FERRI, 2013). Abbiamo notato che la quasi totalità dei docenti predilige un'ampia scelta di tecnologie e strumenti per l'insegnamento della lingua italiana, utilizzando materiale cartaceo, Internet, video e audio quasi nella stessa percentuale. Abbiamo collegato e analizzato i dati emersi dai tre questionari alla domanda riguardante quale materiale avessero utilizzato per insegnare la lingua italiana e ne è emersa una discreta continuità di uso dei diversi materiali: la percentuale maggiore va al supporto cartaceo, come era prevedibile visto il diffuso uso del manuale in quasi tutte le classi. Riguardo al materiale audio, video, Internet, cd rom e alle esperienze nella città, le risposte mostrano che vengono utilizzati dai docenti in percentuali significative. 
Grafico 6 - Materiale utilizzato

\section{Nei tuoi percorsi di insegnamento della lingua italiana quali tra questi materiali utilizzi di solito?}

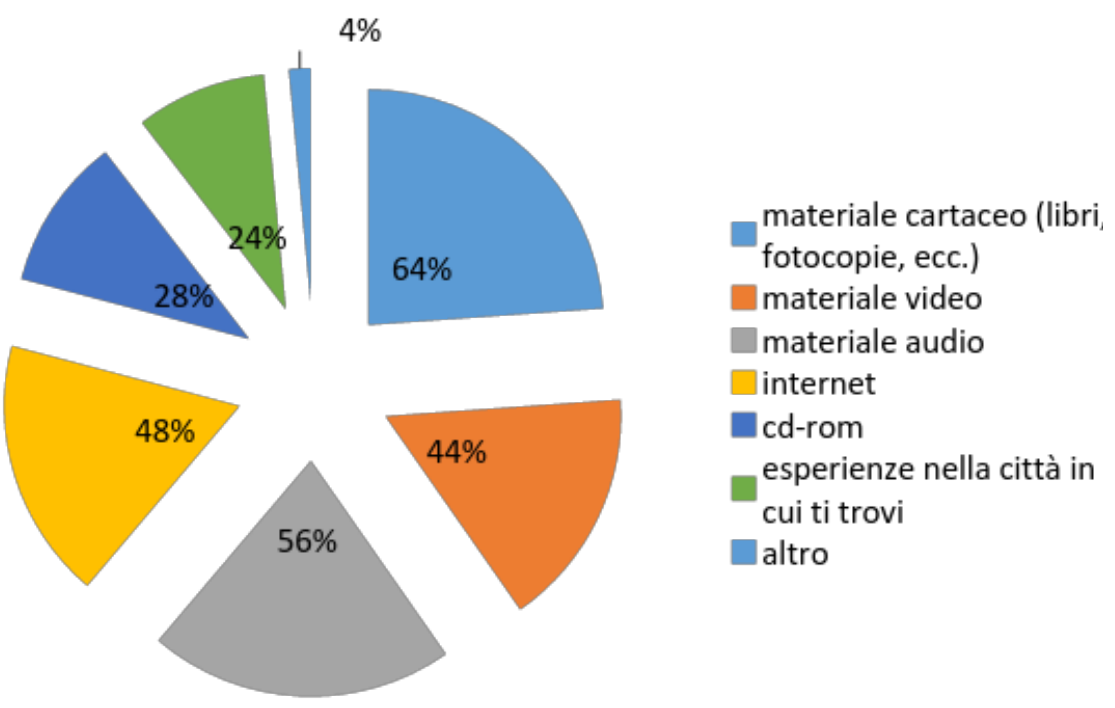

Il grafico ci permette di interpretare i dati in maniera globale: i docenti dei quattro Istituti utilizzano per l'insegnamento in classe della lingua italiana soprattutto materiale cartaceo (64\%), ma vi affiancano tutti gli strumenti che hanno a disposizione: materiale video (44\%), materiale audio (56\%), Internet (48\%), cd-rom (28\%) e esperienze nella città (24\%).

L'uso dell'insieme di tutti questi strumenti nei percorsi di insegnamento ci consente di considerare che il fatto di avere un unico strumento, un ipermedia, in grado di unirli e raccordarli, potrebbe rappresentare una soluzione ottimale per l'insegnamento della lingua.

L'utilizzo di Internet, da parte dei docenti dei quattro Istituti, si estende a tutte le funzionalità proposte con una prevalenza dedicata al realizzare ricerche. L' $88 \%$ indica l'uso di YouTube, il 60\% i giornali on-line, il 48\% giochi e il 20\% il Web Chat. Da questi dati possiamo dedurre che l'integrazione del manuale cartaceo avviene con tutte le potenzialità tecnologiche: l'uso di immagini statiche o in movimento, l'uso di file audio, ricerche ipermediali anche attraverso giornali on-line e Web Chat. 
Grafico 7 - Uso di internet

Nei tuoi percorsi di insegnamento della lingua italiana utilizzi Internet per:

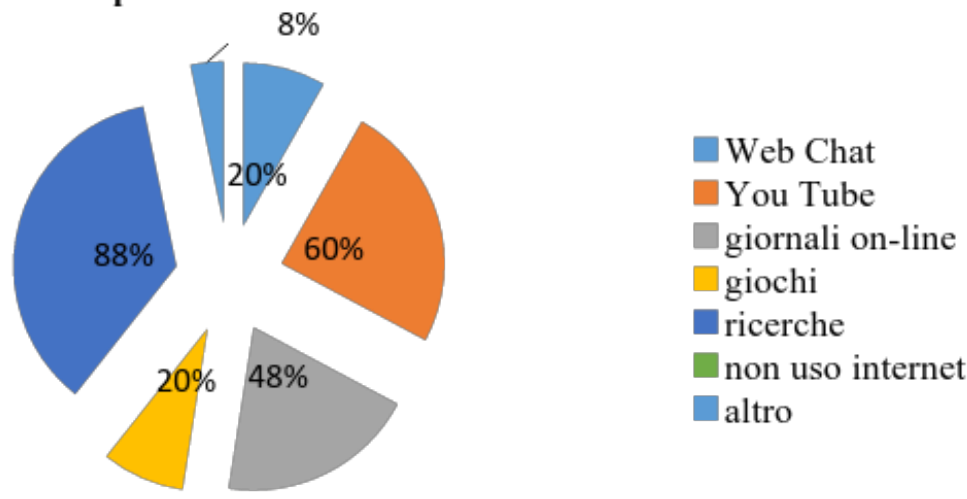

I dati indicati dai docenti sull'efficacia della risorsa Internet in campo glottodidattico è significativa: sottolinea le potenzialità del web come risorsa, senza della quale risulta oggi difficile integrare il manuale di classe. I docenti trovano, tuttavia, poco efficaci i giochi e le Web Chat in rete e attribuiscono a queste due categorie rispettivamente il $24 \%$ e il $20 \%$, mentre considerano più efficaci nella loro pratica didattica YouTube, giornali on-line e ricerche che si assestano tra il $56 \%$ e il $60 \%$.

Grafico 8 - Efficacia degli strumenti disponibili in rete

Quali ti sembrano più efficaci per l'apprendimento della lingua?

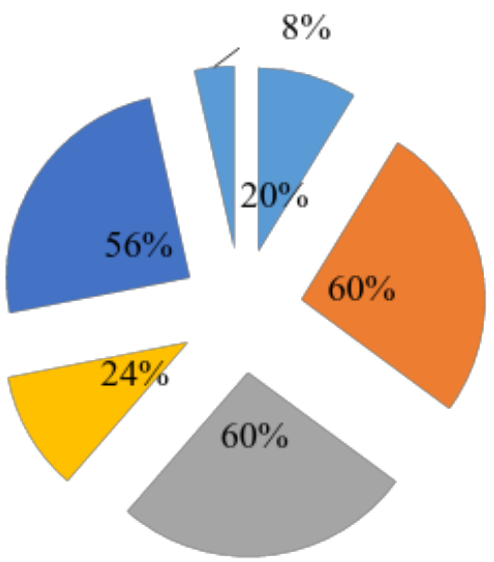

Web Chat

You-Tube

giornali on-line

giochi

ricerche

non uso internet

altro 
L'uso che potrebbe fare il docente della città nella letteratura di riferimento e negli spazi fisici che la città stessa mette a disposizione verrebbe tematizzato e didattizzato nell'ipermedia stesso. Il tema della città andrebbe a coniugare l'esigenza fisica e conoscitiva di attraversare la città fisicamente e costituirebbe uno stimolo all'insegnamento e all'apprendimento. I risultati emersi dal questionario docenti dalle domande riguardanti in modo specifico l'uso della città ci hanno notevolmente sorpresi: non avevamo previsto infatti che anche i docenti di una megalopoli includessero gli spazi urbani nelle loro strategie di insegnamento.

Quando si insegna in un contesto di LS non è così semplice inserire il contatto con la città tra le potenzialità da usare. Va detto però che i docenti con cui siamo entrati in contatto e che operano in realtà europee più gestibili e più relazionate alla lingua italiana, Parigi o Lisbona ad esempio, ne hanno dimostrato la fattibilità. La nostra esperienza di ricerca a San Paolo ha palesato in tutta la sua evidenza il fatto che, trattandosi di una megalopoli, ha tempi di spostamenti molto lunghi. Da ciò avevamo supposto, sbagliandoci, che fossero delle eccezioni le uscite nella città come parte della pianificazione per l'apprendimento della lingua. Come si è detto, a San Paolo, come in molte altre città del mondo, la presenza artistica dell'Italia è notevole. Emerge così dal questionario docenti che questa realtà viene sfruttata per l'insegnamento della lingua e della cultura italiana e dunque che le intersezioni con la lingua e la cultura italiana non sono affatto difficili da trovare e strutturare in ambito glottodidattico.

Infine ci sembra utile analizzare le risposte di tutti i docenti alla domanda che riguardava la pratica dell'insegnamento linguistico e indagava se il docente ricorre al lavoro di gruppo basato sul cooperative learning e/o a gruppi di lavoro non strutturati, al lavoro in coppia e al lavoro individuale.

I docenti dichiarano che in classe ricorrono spesso al cooperative learning, ma anche in ugual misura alle altre strutturazioni della classe. Le risposte alla domanda sull'efficacia evidenziano alcune differenze sull'effettiva pratica: il cooperative learning è in assoluto la struttura di classe considerata più efficace, a discapito del lavoro in gruppi non strutturati, del lavoro a coppie e a quello individuale. Possiamo ipotizzare, in questa sede di analisi, che ci sia stata una riflessione da parte dei docenti al momento di rispondere alla parte del questionario sugli approcci che mettono in atto e sulla loro efficacia. 
Grafico 9 - Suddivisione della classe durante le lezioni

Nell'insegnamento della lingua italiana tu utilizzi:

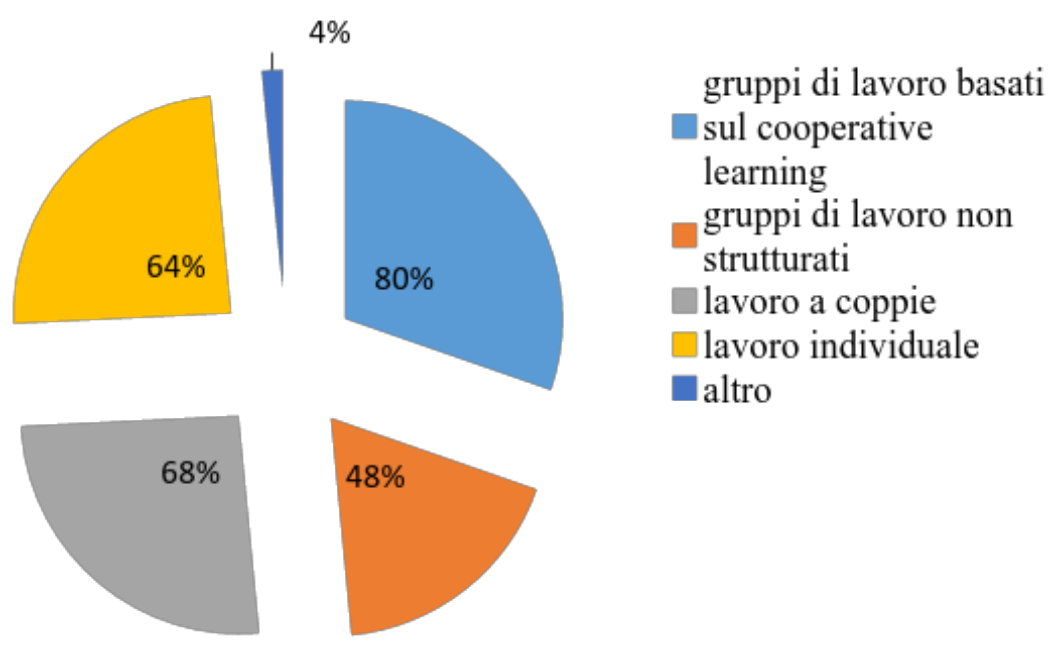

Grafico 10 - Efficacia delle diverse forme di suddivisione della classe durante le lezioni

Quali ti sembrano più efficaci?

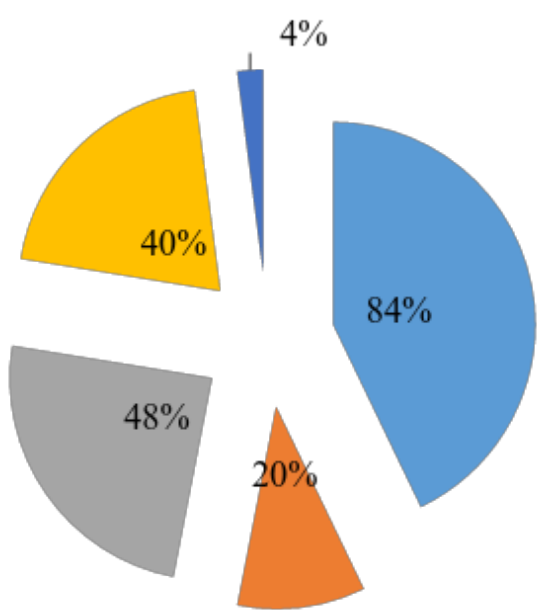

gruppi di lavoro basati

sul cooperative learning gruppi di lavoro non strutturati

lavoro a coppie $\square$ lavoro individuale altro 


\section{Una conclusione}

La concisa presentazione dei dati e la loro analisi esposta nei paragrafi precedenti ci inducono al tentativo di osservarli da un punto di vista più globale. Guardando i risultati del questionario docenti e del questionario studenti, emerge innanzitutto la questione dell'età: la maggior parte delle persone che ha risposto è in una fascia di età che la esclude dall'esser nata in periodo digitale. Ciononostante, tanto i docenti quanto gli studenti, in ambito di insegnamento e apprendimento delle lingue, utilizzano sempre molti degli strumenti che la tecnologia mette a disposizione. Le potenzialità di Internet, presente in tutti gli Istituti presi in esame, vengono infatti sfruttate indistintamente da docenti e studenti in ambito glottodidattico. Pensiamo che una percentuale così alta di persone che si rapportano con strumenti tradizionali e strumenti tecnologici in classe sia una caratteristica della classe di lingua.

Una seconda osservazione riguarda la città come strumento per insegnare e apprendere la lingua: le risposte ai questionari ci rivelano come la città venga inserita nei percorsi istituzionali di apprendimento e in quelli autonomi. È interessante notare come la città venga sfruttata non solo nelle visite ai musei, ma anche negli spazi pubblici che mette a disposizione, nonché nella partecipazione a incontri e dibattiti.

Abbiamo, infine, trovato di un certo rilievo il fatto che sia studenti che docenti propendano per lavori di gruppo in classe, sia in cooperative learning che non strutturati, o anche lavoro a coppie.

Pertanto, alla luce di tutti i dati commentati possiamo osservare come le caratteristiche che avremmo attribuito solo ai "nativi digitali" siano in realtà perlopiù caratteristiche comuni anche alle persone nate molto prima dell'inizio dell'era digitale: da una parte, soggetti che studiano la lingua e, dall'altra, specularmente, soggetti che insegnano la lingua con strumenti e modalità che sono un ponte tra l'apprendimento formale e l'apprendimento informale

Come già evidenziato l'obiettivo della macro-ricerca è quello di analizzare i vari fattori che inducono a pensare l'utilizzo di un ipermedia nella didattica della lingua come evoluzione glottodidattica rispetto al tradizionale manuale. La ricerca presentata si è posta come obiettivo osservare nel dettaglio le pratiche più diffuse nell'insegnamento e nell'apprendimento della lingua italiana, soprattutto per quanto riguarda l'uso di materiali e strumentazioni multimediali. Le risposte vanno tutte in direzione ipermediale, dunque dell'utilizzo da parte di tutti gli intervistati di più supporti multimediali in ambito glottodidattico.

In questo contesto presupponiamo che l'utilizzo di un ipermedia nella classe di italiano, come sostitutivo del libro di testo, possa essere uno strumento completo, un ponte tra la tradizione e il futuro, tra il cartaceo e le tecnologie. 


\section{Riferimenti bibliografici}

ACUTIS, C., Insegnare letteratura, Roma: Pratiche, 1979.

ANTONELLI, G., Lingua ipermedia, Lecce: Manni, 2002.

BALBONI, P.E. (a cura di), Educazione letteraria e nuove tecnologie, Torino: Utet Libreria, 2004.

BALDRY, A.; PROZZO, N., Ipermedia nell'educazione linguistica, Roma: Armando Editore, 1996.

BANZATO, M., Apprendere in rete, Torino: Utet Libreria, 2002.

; CORCIONE, D., Tecnologie educative, Bologna: CLUEB, 2007.

BURGESS, R.G. (a cura di), Issues in Educational Research: Qualitative Methods. London: Falmer, 1985.

CADAMURO, A., Stili cognitivi e stili di apprendimento, Roma: Carocci, 2004.

CAON, F. (a cura di), Insegnare italiano nella classe ad abilità differenziate. Perugia: Guerra, 2006. , Educazione linguistica e differenziazione, Torino: Utet Università, 2008.

CIOTTI, F.; RONCAGLIA, G. Il mondo digitale, Bari: Laterza, 2000.

CLARK, R.C.; LYONS, C., Graphics for Learning: Proven Guidelines for Planning, Designing, and Evaluating Visuals in Training Materials, San Francisco: Pfeiffer, 2000.

COONAN, C., La ricerca-azione, Venezia: Libreria Editrice Cafoscarina, 2000.

FERRARI, S., Le tecnologie digitali per l'educazione linguistica, Milano: EDUCatt, 2012.

FERRI, P., Nativi digitali, Milano: Bruno Mondadori, 2011.

. La scuola 2.0. Verso una didattica aumentata dalle tecnologie, Parma: Casa Editrice

Spiaggiari, 2013.

GARDNER, H., Multiple Intelligences: The Theory in Practice, New York: Basic Book, 1993. ,Educazione e sviluppo della mente, Trento: Erickson, 2005.

LODRINI, T. (a cura di), Didattica costruttivista e ipermedia, Milano: Franco Angeli, 2002.

MORGAN, D.L., Focus groups as qualitative research. $2^{\text {nd }}$ ed. London: Sage, 1997.

PRENSKY, M., Digital Natives, Digital Immigrants, On the Horizon, Vol. 9, nn. 5-6, 2001.

PICHIASSI, M., Apprendere l'italiano L2 nell'era digitale. Le nuove tecnologie nell'insegnamento e apprendimento dell'italiano per stranieri, Perugia: Guerra Edizioni, 2007.

TOSCANO, A. Letteratura, città, web. In: CAON, F.; SERRAGIOTTO, G. (a cura di), Tecnologia e didattica delle lingue, Torino: Utet Università, 2012.

TOSCANO, A., Attività fuori aula e tecnologie ipermediali nella didattica della lingua italiana. Innovation in Methodology and Practice in Language Learning, Newcastle upon Tyne: Cambridge Scholars Publishing, 2015.

Recebido em 10/08/2016

Aprovado em 15/11/2016 